\title{
Impact of Vibrational Modes in the Plasmonic Purcell Effect of Organic Molecules
}

\author{
Dongxing Zhao, Rui E. F. Silva, Clàudia Climent, Johannes Feist, Antonio I. Fernández-Domínguez, \\ and Francisco J. García-Vidal*
}

Cite This: ACS Photonics 2020, 7, 3369-3375

Read Online

ABSTRACT: By means of quantum tensor network calculations, we investigate the large Purcell effect experienced by an organic molecule placed in the vicinity of a plasmonic nanostructure. In particular, we consider a donor- $\pi$ bridge-acceptor dye at the gap of two Ag nanospheres. Our theoretical approach allows for a realistic description of the continua of both molecular vibrations and optical nanocavity modes. We analyze both the ultrafast exciton dynamics in the large Purcell enhancement regime and the corresponding emission spectrum, showing that these magnitudes are not accurately represented by the simplified models used up to date. Specifically, both the two-level system model and the single vibrational mode model can only reproduce the

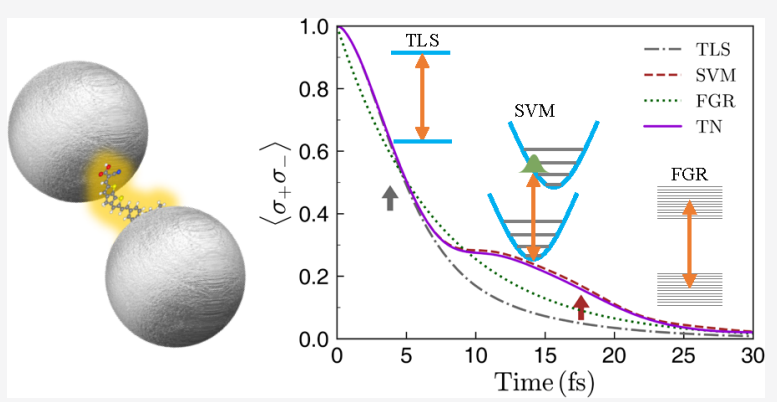
dynamics over short time scales, whereas the Fermi's golden rule approach accounts only for the behavior at very long times. We demonstrate that including the whole set of vibrational modes is necessary to capture most of the dynamics and the corresponding spectrum. Moreover, by disentangling the coupling of the molecule to radiative and nonradiative plasmonic modes, we also shed light into the quenching phenomenology taking place in the system.

KEYWORDS: excited-state dynamics, emission spectrum, tensor networks, two-level system, single vibrational mode model, Fermi's golden rule

T he Purcell effect ${ }^{1}$ lies at the core of quantum electrodynamics, as it reveals that the radiative properties of any quantum emitter are not inherent to it, but also depend on the electromagnetic (EM) vacuum in its surroundings. A few decades ago, this phenomenon, and in particular, the pursuit for the full inhibition of spontaneous emission at EM band gaps, was one of the driving forces behind the development of photonic crystals. ${ }^{2}$ More recently, much interest has focused on metallic nanocavities. ${ }^{3,4}$ The large and spectrally complex photonic density of states associated with plasmonic resonances allows an unprecedented control over spontaneous emission in these nanostructures. ${ }^{5,6}$ In recent years, different light sources have been used to probe plasmonic Purcell enhancement phenomena, such as quantum dots, ${ }^{7,8}$ solid-state color centers ${ }^{9,10}$ or passing electron beams. ${ }^{11,12}$

Due to their large transition dipole moments and stability at room temperature, organic molecules have received increasing attention as reliable quantum emitters. In their interaction with plasmonic nanostructures, several topics have been addressed, such as fluorescence enhancement, ${ }^{13-17}$ spectral shaping, ${ }^{18-25}$ and strong coupling. ${ }^{26-32}$ Unlike other microscopic light sources, for which a two-level system (TLS) description is usually accurate, electronic transitions of organic molecules, from now on excitons, interact strongly with the molecular nuclear vibrations.
Within the TLS approach, only a few time scales play an important role, and in particular, the relative values of the plasmon-exciton exchange rate and their respective losses can be used to distinguish between the weak-coupling and strongcoupling regimes in a fundamental analysis. In contrast, in molecules, the vibronic coupling and the vibrational dynamics introduce several new time scales, such as the coherent nuclear oscillation period or the rate of vibrational energy dissipation and thermalization time, and the simple dichotomy between weak and strong coupling can be expected to give way to a richer phenomenology. For the case of not too large Purcell factors, radiative decay is typically slower than the vibrational relaxation and thermalization times (on the order of 1 ps or less ${ }^{33}$ ), and molecular emission can be assumed to proceed from the lowest excited molecular state, with all vibrational modes in thermal equilibrium. This assumption gives rise to a Fermi's golden rule (FGR)-based approach in which the emission spectrum of the organic molecule near a plasmonic

Received: July 9, 2020

Published: November 30, 2020 
structure is given by the product of its free-space spectrum and a frequency-dependent Purcell enhancement. ${ }^{19,20,34}$ However, as plasmonic structures can achieve Purcell factors on the order of $10^{6}{ }^{29}$ radiative decay rates can be decreased from their freespace values in the nanosecond range to femtoseconds, such that radiative decay does not proceed from vibrationally relaxed molecules, the FGR approximation breaks down, and nonequilibrium effects play an important role. More recently, cavity-QED approaches have been able to incorporate the interplay between the exciton and one (or a few) vibrational modes. ${ }^{35-39}$ However, a realistic description of typical organic molecules could require considering hundreds of vibrational modes.

In this Article we present an accurate theoretical framework that is able to treat on an equal footing the electronic, vibrational, and plasmonic degrees of freedom associated with the Purcell effect experienced by organic molecules placed within a metallic nanocavity. Our numerical scheme is based on a quantum tensor network (TN) method, ${ }^{40,41}$ which helps provide a complete picture of this phenomenon. In addition, it allows for a validation of the simple models introduced above and also for an in-depth study of the influence of the large number of vibrational modes on the spontaneous emission rate and emission spectrum. We show that while the TLS model is able to reproduce the very short time scales of the spontaneous decay, the FGR approach accounts only for the behavior at very long times. On intermediate time scales, a model that incorporates a single vibrational mode is able to extend the validity of the TLS to slightly longer times. However, we demonstrate that it is mandatory to account for the whole set of vibrational modes to capture most of the time dynamics and the corresponding spectrum.

Model. As a case study, we consider the excited-state dynamics of a single organic molecule placed at the gap center of a silver nanosphere dimer, as sketched in the inset of Figure 1a. This plasmonic structure resembles the two geometries in which the largest Purcell factors have been reported, the socalled nanoparticle on mirror ${ }^{4}$ and bowtie antenna ${ }^{16}$ geometries. In this work we have chosen a donor- $\pi$ bridgeacceptor (D- $\pi$-A) organic dye, labeled $\mathrm{CPDT}^{42}$ as a prototypical organic molecule because it displays a significant transition dipole moment and a large Stokes shift $(>0.4 \mathrm{eV})$, yielding excellent absorption and emission capabilities. The Hamiltonian for this hybrid system can be written as $[\hbar=1]$

$$
\begin{aligned}
H= & \omega_{e} \sigma_{+} \sigma_{-}+\int \mathrm{d} \omega\left[\omega b_{\omega}^{\dagger} b_{\omega}+\lambda_{\omega}\left(b_{\omega}^{\dagger}+b_{\omega}\right) \sigma_{+} \sigma_{-}\right] \\
& +\sum_{i=\mathrm{nr}, \mathrm{r}} \int \mathrm{d} \omega\left[\omega a_{i, \omega}^{\dagger} a_{i, \omega}+g_{i, \omega}\left(a_{i, \omega}^{\dagger} \sigma_{-}+a_{i, \omega} \sigma_{+}\right)\right]
\end{aligned}
$$

where $\sigma_{-}, b_{\omega}$, and $a_{i, \omega}$ denote the lowering operator of the molecular electronic transition, annihilation operator for the molecular vibration, and $i$-component of the plasmonic mode at frequency $\omega$. Here the index $i$ is either $\mathrm{nr}$ or $\mathrm{r}$, representing the nonradiative and radiative plasmonic modes, respectively. Notice that only the latter can be detected in the far-field. The first line in eq 1 describes the free-standing molecule, where we have used a Holstein-type Hamiltonian ${ }^{43}$ accounting for the continuum of vibrational modes, with coupling strengths $\lambda_{\omega}$. The exciton frequency $\omega_{e}$ corresponds to the vertical transition from the vibrational ground state of the electronic ground state. The last part in eq 1 describes the continuum of radiative and nonradiative plasmonic modes, and their coupling, weighted by $g_{i, \omega}$, to the molecular exciton. This plasmon-
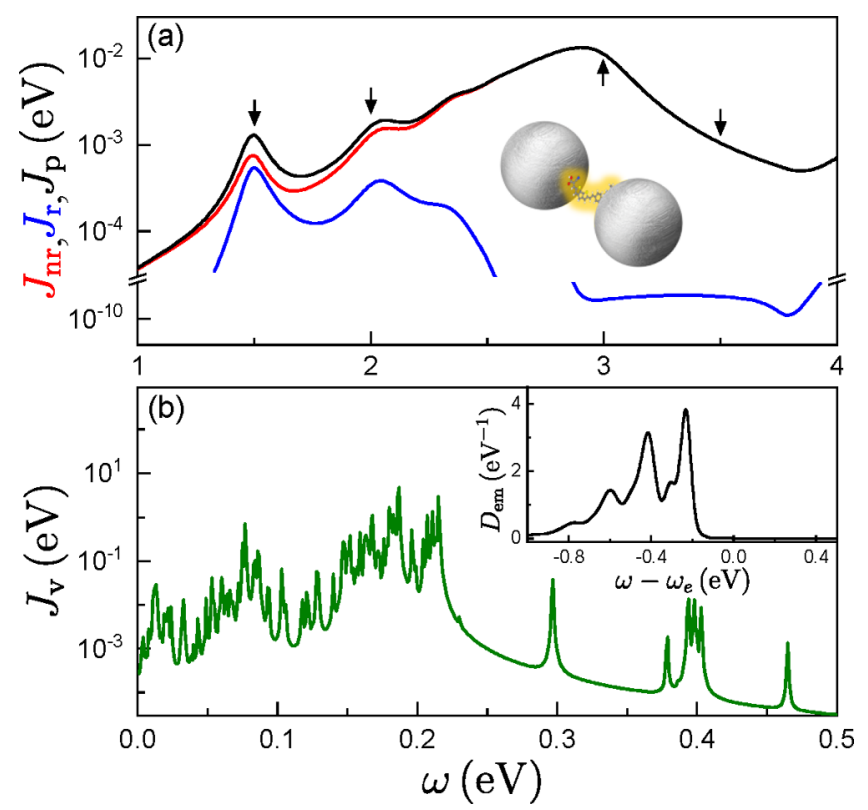

Figure 1. (a) Radiative $(i=\mathrm{r}$, blue) and nonradiative $(i=\mathrm{nr}$, red) plasmonic spectral densities, evaluated at the gap center of a nanosphere dimer, sketched as an inset. The black curve shows the total spectral density, $J_{\mathrm{p}}(\omega)$, while the arrows indicate the exciton frequencies in Figures 2 and 3. (b) Vibrational spectral density, $J_{\mathrm{v}}(\omega)$, for the CPDT molecule. The inset shows the line shape function, $D_{\mathrm{em}}(\omega)$, at $T=300 \mathrm{~K}$.

exciton coupling can be encoded in the spectral density $J_{i}(\omega)=$ $g_{i, \omega}^{2}$, in a similar way as the vibrational spectral density $J_{\mathrm{v}}(\omega)=$ $\lambda_{\omega}^{2}$ does for the vibronic coupling. Note that eq 1 includes optomechanical coupling, ${ }^{44}$ which is usually described in a form where the exciton operators have been adiabatically eliminated. More details of the theoretical model can be found in the Supporting Information, which includes refs 45-57.

We numerically solve both the excited-state dynamics and the emission spectrum with our quantum TN method. Importantly, this simulation tool applies a variational algorithm to describe the dynamical evolution of the system wave function. This is expressed as a tensor network, whose truncation must be carefully treated to ensure convergence. ${ }^{48,51}$ To make the system described by eq 1 amenable to treatment by this method, the two continua are transformed to a chain form in which each continuum (EM and vibrational) is represented by a chain of nearest-neighbor coupled oscillators, with only the first oscillator coupled to the exciton (see Supporting Information for more details of $\mathrm{TN}$ calculations). The first site in the vibrational chain is usually termed the reaction coordinate (RC), whose frequency is given by

$$
\omega_{\mathrm{RC}}=\frac{1}{\lambda_{\mathrm{RC}}^{2}} \int \omega J_{\mathrm{v}}(\omega) \mathrm{d} \omega
$$

with $\lambda_{\mathrm{RC}}=\sqrt{\int J_{\mathrm{v}}(\omega) \mathrm{d} \omega}$ being the coupling strength between the RC and the exciton. The remaining sites in the chain essentially act as an effective bosonic environment that leads to the vibrational decay and dephasing of the RC itself.

To shed light into the effect of molecular vibrations and uncover the relevant time scales in the spontaneous emission process, we compare our TN numerical results against three simplified models. As the simplest choice, by discarding all the 
molecular vibrations, the standard TLS model predicts a decay only dictated by the plasmonic environment, $\gamma_{\mathrm{TLS}}=2 \pi J_{\mathrm{p}}\left(\omega_{e}\right)$, where $J_{\mathrm{p}}(\omega)=\sum_{i} J_{i}(\omega)$ is the total plasmonic spectral density. In a second step, by keeping only the $\mathrm{RC}$ within the vibrational chain, we can derive an approach, dubbed here as the single vibration mode (SVM) approximation, in which the RC comprises all the vibrational response. Finally, within the FGR model (see Supporting Information for details), the spontaneous emission rate is simply calculated as the spectral integral of the product of $J_{\mathrm{p}}(\omega)$ with the so-called line shape function, $D_{\mathrm{em}}(\omega)$, which represents the available optical transitions connecting the ground and the excited electronic states of the molecule under the assumption that the vibrational modes are in thermal equilibrium, leading to

$$
\gamma_{\mathrm{FGR}}=2 \pi \int \mathrm{d} \omega D_{\mathrm{em}}(\omega) J_{\mathrm{p}}(\omega)
$$

As commented above, we consider a silver nanosphere dimer as an example plasmonic cavity, with a $1 \mathrm{~nm}$ gap and $20 \mathrm{~nm}$ radius. It is embedded in a matrix with refractive index $n_{\mathrm{D}}=$ 2.1, which models a DNA scaffolding. ${ }^{32}$ The dielectric constant for silver is taken from ref 58. Numerical EM simulations were performed using the finite-element solver of Maxwell's equations implemented in the commercial package COMSOL Multiphysics. Radiative and nonradiative contributions to the Purcell factor and spectral density were disentangled using the approach in ref 59. As shown in Figure 1a, the plasmonic spectral density is characterized by a radiative dipole mode located at around $1.5 \mathrm{eV}$, a quadrupole mode at $2.1 \mathrm{eV}$, and a dominant nonradiative mode, the so-called pseudomode, ${ }^{60}$ emerging at $2.9 \mathrm{eV}$. A single CPDT molecule is located at the gap center, and we assume that its transition dipole moment (modulus $\mu=0.1 e \cdot n m$ ) is pointing along the line that connects the two nanospheres. In the numerical calculations, we assume that the initial state originates from a FranckCondon excitation, that is, a vertical transition that could result after an ultrashort laser pulse excitation of the electronic ground state. The line shape function within the FGR model in the main text is calculated at $T=300 \mathrm{~K}$, and a comparison with pseudo temperatures simulating the Franck-Condon distribution is provided in the Supporting Information. The temperature in the TN and SVM calculations is effectively zero, as it does not play a relevant role on the ultrashort time scales that we are investigating in this work.

Electronic structure calculations within density functional theory and its time-dependent version are performed to obtain the vibronic coupling constants of the CPDT dye for the displaced harmonic oscillator model. Both the vibrational spectral density and line shape function of the CPDT molecule used in our calculations are shown in Figure 1b. In order to explore different regions of the plasmonic spectral density, we artificially shift the (vertical) exciton energy of the molecule to different values while keeping its vibrational structure fixed. This permits the study of the influence of the different features in the plasmonic spectral density on the molecular dynamics without using molecules with different excitation energies and a different vibrational structure. Therefore, our model should be considered as an archetype for an organic molecule with a complex vibrational spectrum and a large exciton-phonon coupling.

\section{RESULTS AND DISCUSSION}

We focus first on the excited-state dynamics. Figure 2 shows the evolution of the exciton population, $\left\langle\sigma_{+} \sigma_{-}\right\rangle$, evaluated with

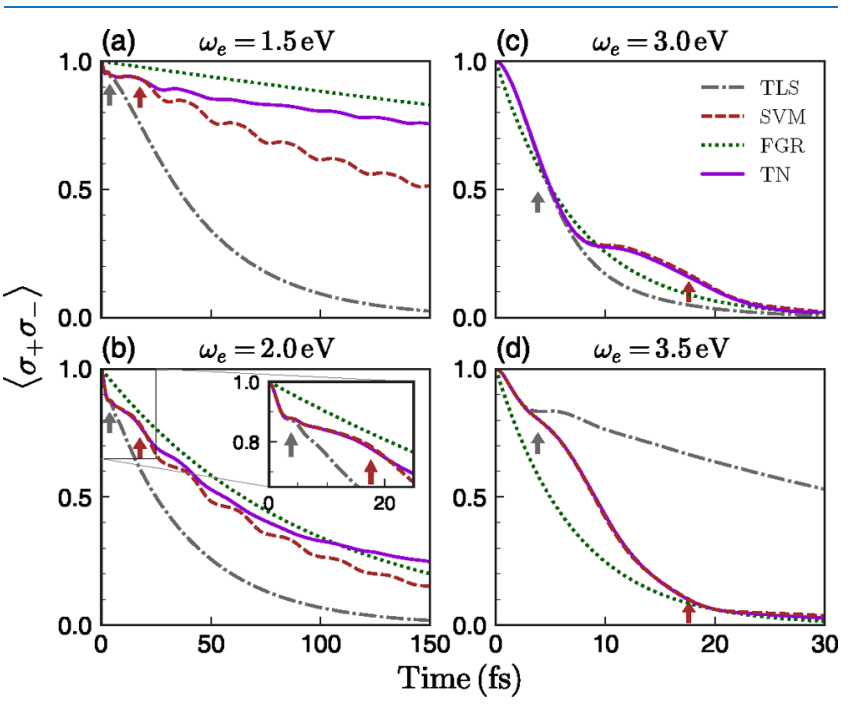

Figure 2. Excited-state dynamics $\left\langle\sigma_{+} \sigma_{-}\right\rangle$vs time for different exciton frequencies. In each panel, TLS (gray dash-dotted), SVM (brown dashed), FGR (green dotted), and exact TN (violet solid) predictions are shown. The arrows indicate the positions of $\tau_{1} \approx \pi /\left(3 \omega_{\mathrm{RC}}\right)$ (gray) and $\tau_{2}=1 / \gamma_{\mathrm{d}}$ (brown), with $\omega_{\mathrm{RC}}$ and $\gamma_{\mathrm{d}}$ being defined in eqs 2 and 4 , respectively. The inset of panel (b) shows a zoom at a shorter temporal range.

the TN method at four exciton frequencies. It is compared against the results of the three different models (TLS, SVM, and FGR) for the same cases. First, it is evident that the TLS model largely fails to describe the decay dynamics for all the cases depicted in Figure 2. This failure highlights the importance of going beyond the TLS approach when defining the coupling regimes in the interaction of organic molecules with nanophotonic structures, implying that the simple distinction between weak and strong coupling has to be modified for organic molecules. More specifically, the onset of strong coupling has been identified with the emergence of (Rabi) oscillations in the excited state population dynamics. This picture emerges from a TLS model for the molecule. Our results include molecular features, such as the coherent nuclear oscillation or the rate of vibrational energy dissipation, which also yield an oscillatory behavior of the exciton population even in the Purcell regime, as discussed below. Still, it is interesting to note that TLS seems to be valid up to times of about $\tau_{1} \approx 4$ fs (gray arrows in Figure 2). This is because, soon after its generation, the exciton vibrational wavepacket remains in the vicinity of the Franck-Condon region before exploring the potential energy surface of the excited state. ${ }^{61}$ Hence, within a very short time scale, vibrations do not play any role yet and the TLS model describes the molecular decay. This time scale, $\tau_{1}$, can be estimated as a fraction of the period associated with the RC harmonic motion, $T_{\mathrm{RC}}=2 \pi / \omega_{\mathrm{RC}}$. Specifically, $\tau_{1}$ must be shorter than $T_{\mathrm{RC}} / 4$, which is the time that the vibrational wavepacket takes to reach the equilibrium position in the potential energy surface for the excited electronic state. We find that $\tau_{1} \approx T_{\mathrm{RC}} / 6=3.8 \mathrm{fs}$, which is in very good agreement with our numerical results.

When the wavepacket initially moves away from the Franck-Condon region, its dynamics is dominated by the 
$\mathrm{RC}$, as revealed by the accuracy of the SVM description for all $\omega_{e}$ in Figure 2. This regime holds until both dephasing and decay of the RC into other sites in the $\mathrm{TN}$ vibrational chain becomes important. We can estimate this second time scale, $\tau_{2}$, as the inverse of the coupling between the first (RC) and second sites of the vibrational chain,

$$
\gamma_{\mathrm{d}}=\sqrt{\frac{1}{\lambda_{\mathrm{RC}}^{2}} \int \omega^{2} J_{\mathrm{v}}(\omega) \mathrm{d} \omega-\omega_{\mathrm{RC}}^{2}}
$$

This estimation gives $\tau_{2}=1 / \gamma_{\mathrm{d}}=17.6 \mathrm{fs}$ (brown arrows in Figure 2). This time scale sets the validity of the SVM treatment, and explains that the excellent agreement with TN calculations at high $\omega_{e}$ resides in the large plasmonic coupling experienced by the molecule in this regime.

Following these arguments, it can be understood why the FGR model, in which all vibrational modes are taken into account, works better than the TLS and SVM models at sufficiently long times, as observed in Figure 2. Importantly, it reproduces the general slope of the $\mathrm{TN}$ exciton dynamics at long times, which implies a valid estimate of the Purcell factor experienced by the molecule. However, FGR assumes that the vibrational modes at all times are in thermal equilibrium and thus neglects the initial strongly nonequilibrium state and its coherent wave packet motion. The FGR approach thus fails to capture the short-time dynamics and, in particular, cannot represent the weak oscillations observed in the $\mathrm{TN}$ calculations.

Let us compare quantitatively the plasmonic Purcell factors that we obtain from the TLS and FGR models at the four exciton frequencies marked by vertical arrows in Figure 1a. The former is given by $J_{\mathrm{p}}\left(\omega_{e}\right)$ normalized by its free space counterpart, $J_{0}\left(\omega_{e}\right){ }^{60}$ The latter results from the ratio between $\gamma_{\mathrm{FGR}}$ and the free-space decay rate obtained by replacing $J_{\mathrm{p}}(\omega)$ by $J_{0}(\omega)$ in eq 3 . At $\omega_{e}=1.5 \mathrm{eV}$, the TLS Purcell factor is $2.0 \times$ $10^{6}$, while the FGR approximation yields $2.5 \times 10^{5}$, almost 1 order of magnitude apart. Note that, at this exciton frequency, the emission in the TLS is at resonance with the dipolar plasmon mode, while the FGR is Stokes-shifted with respect to it. The discrepancy between TLS $\left(1.1 \times 10^{6}\right)$ and FGR $(7.1 \times$ $10^{5}$ ) factors is significantly lower at $\omega_{e}=2.0 \mathrm{eV}$, due to the impact of the dipolar plasmon on the FGR model at this exciton frequency. At $\omega_{e}=3.0 \mathrm{eV}$, both approximations are in very good agreement $\left(2.1 \times 10^{6}\right)$, a direct consequence of the broad nature of the plasmonic pseudomode sustained by the nanosphere dimer. Finally, the TLS $\left(1.3 \times 10^{5}\right)$ predicts a much slower decay than the FGR $\left(1.3 \times 10^{6}\right)$ above the pseudomode $\left(\omega_{e}=3.5 \mathrm{eV}\right)$.

We next examine the frequency-dependent population of the EM modes, $S_{\mathrm{em}}(\omega)=\sum_{i}\left\langle a_{i}^{\dagger}(\omega) a_{i}(\omega)\right\rangle$. Notice that the sum extends over both radiative and nonradiative plasmonic modes, so we name this physical magnitude the near-field emission spectrum. Within the FGR approach, this quantity is independent of time and can be written as $S_{\text {em }}(\omega) \propto$ $D_{\mathrm{em}}(\omega) J_{\mathrm{p}}(\omega)$, which gives an intuitive relation between emission peaks and EM spectral density for organic molecules. Figure 3 shows the near-field emission spectra calculated for the four exciton frequencies and the four theoretical approaches in Figure 2, evaluated at $t=150$ fs (see Supporting Information for spectra evaluated at the initial and intermediate stage). It is worth pointing out that this corresponds to the total emission accumulated up to this point in time, not an instantaneous emission spectrum. First

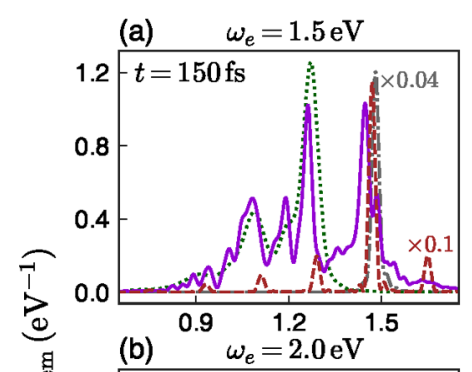

(c) $\omega_{e}=3.0 \mathrm{eV}$


(d)

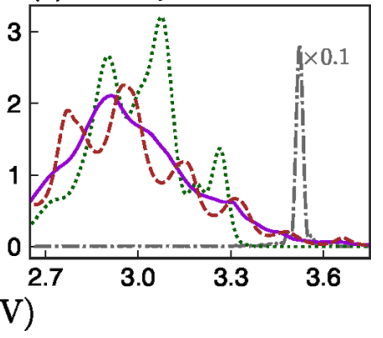

Figure 3. Near-field emission spectra $S_{\mathrm{em}}(\omega)$ for four different exciton frequencies. The spectra for the TLS and SVM approximations and full TN method are calculated at $t=150 \mathrm{fs}$. The line code is the same as in Figure 2. Some spectra have been scaled to facilitate comparison.

note that in the left and right panel of Figure 3, emission peaks located at 1.5 and $2.9 \mathrm{eV}$ are clearly seen in $\mathrm{TN}$ results, indicating that they are closely related to the dipole mode and pseudomode, respectively. Within the TLS model, only the EM modes close to $\omega_{e}$ contribute to the spontaneous emission process. This leads to single-peaked spectra broadened by the plasmonic environment, very different to the spectra obtained within the TN framework. On the other hand, the SVM approximation largely fails to reproduce the TN spectra for low exciton frequencies (1.5 and $2.0 \mathrm{eV}$ ), although it provides a reasonable approximation for higher $\omega_{e}$. This is in accordance with its better accuracy describing the excited-state decay dynamics for those frequencies (see Figure 2).

In principle, one could have expected that the FGR approach should work better than the other two simplified models, as it incorporates the whole vibration spectrum via the line shape function of the organic molecule. However, as observed in Figure 3, this is not the case for the four chosen $\omega_{e}$. The FGR approach only works well when the coupling of the molecular exciton with its EM environment is weak enough such that vibrational thermal equilibrium is reached before emission takes place. However, for large plasmon-exciton couplings leading to huge Purcell factors as those associated with plasmonic fields, the molecular exciton decays so fast that vibrational equilibrium is not reached and the spectrum is significantly different from the stationary limit. Our TN calculations show that this fast decay can be utilized to strongly modify the branching ratio of the emission by organic molecules, a phenomenon that has been also reported experimentally. $^{23,24}$

Finally, taking advantage of the capability of our TN theoretical framework to separate contributions from plasmonic radiative and nonradiative channels, here we address the interplay between the Purcell effect and the quenching phenomenon. Figure 4 shows the evolution of the population of EM modes, $n_{i}=\int \mathrm{d} \omega\left\langle a_{i}^{\dagger}(\omega) a_{i}(\omega)\right\rangle$ ( $i$ being either nr or $\mathrm{r}$ ), as a function of exciton frequency and evaluated at three different times. At very short time scales ( $t=5 \mathrm{fs}$, panel a), the TLS approximation reproduces the TN result, since the 

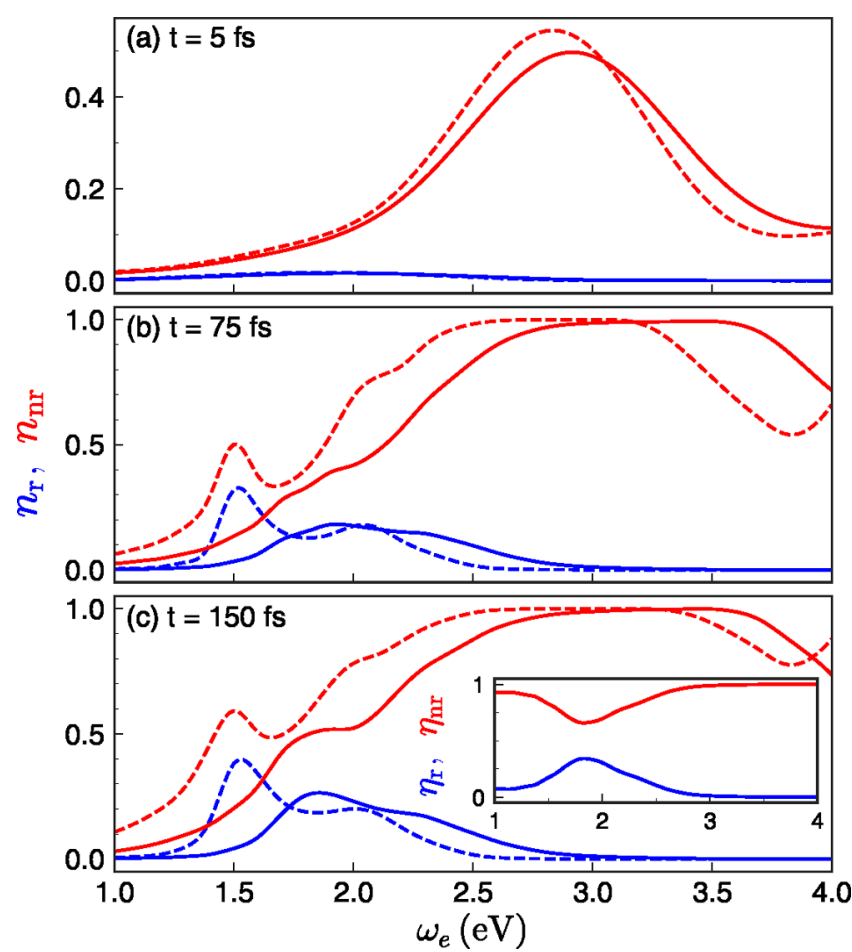

Figure 4. Radiative and nonradiative plasmon population $\left(n_{\mathrm{nr}}\right.$ and $\left.n_{\mathrm{r}}\right)$ as a function of exciton frequency $\omega_{e}$ at (a) $t=5$, (b) 75 , and (c) 150 fs, calculated from the TLS approximation (dashed) and full TN framework (solid). The inset in panel (c) shows the relative contributions of the radiative (effective quantum yield) and nonradiative plasmonic modes.

molecular wavepacket has not yet explored the molecular vibrations. We can observe that only the coupling to the plasmonic pseudomode (around $2.9 \mathrm{eV}$ ) is fast enough to yield a significant EM population in this time scale. The dark nature of this plasmonic resonance translates into a vanishing radiative EM spectrum. The radiative population becomes significant only at longer times, as shown in Figure 4b,c. Specifically, both spectra present a maximum in the vicinity of $1.85 \mathrm{eV}$, which is a result of maximizing the spectral overlap between $D_{\mathrm{em}}(\omega)$ and $J_{\mathrm{r}}(\omega)$ and, hence, slightly blue-detuned from the dipolar plasmon mode frequency $(1.5 \mathrm{eV})$ given by the TLS model. Moreover, note that these two panels are very similar, implying that the coupling rate between the molecule exciton and this bright plasmon resonance is faster than $75 \mathrm{fs}^{-1}$. Within this exciton frequency range, the effective quantum yield, shown in the inset of Figure 4c, can be as high $30 \%$, despite the fact that the gap in the plasmonic cavity is only $1 \mathrm{~nm}$ wide and quenching is expected to be dominant. For higher $\omega_{e}$, nonradiative components take over, completely quenching the far-field emission of photons by the molecule, as expected. By comparison, the TLS approximation only reproduces qualitatively the $\mathrm{TN}$ profiles at longer times, a direct consequence of the discrepancies in the emission spectra in Figure 3.

\section{CONCLUSION}

To conclude, we have applied an accurate quantum tensor network method, able to treat electronic, electromagnetic and vibrational degrees of freedom in light-matter scenarios on an equal footing, to study the Purcell effect occurring when organic molecules interact with plasmonic nanocavities. Using this numerical framework we have tested different simplified models that have been used to analyze this phenomenon lately. We have found that a two-level description of the molecule fairly captures the first steps of the decay process and a simple approach based on the Fermi golden rule can describe its very long time behavior. However, an accurate treatment of the exciton coupling to all the vibrational modes in the organic molecule is mandatory to resolve intermediate time scales. As a consequence, none of the simplified models used up to date are able to provide a precise estimation of near- or far-field emission spectra in hybrid systems involving organic molecules and plasmonic cavities. As an interesting extension of the current work, the influence of nonlinear vibrational mode couplings leading to vibrational energy redistribution could be investigated, as they are not included in the current Holsteintype model and are expected to become important on slightly longer time scales than treated here. ${ }^{62,63}$ Our findings also reveal that significant effective quantum yield values can be achieved in situations where strong interaction to nonradiative plasmonic modes takes place and quenching is expected to dominate.

\section{ASSOCIATED CONTENT}

\section{Supporting Information}

The Supporting Information is available free of charge at https://pubs.acs.org/doi/10.1021/acsphotonics.0c01095.

More details about the theoretical model, tensor network calculations, Fermi's golden rule approach, and time-dependent emission spectra (PDF)

\section{AUTHOR INFORMATION}

\section{Corresponding Author}

Francisco J. García-Vidal - Departamento de Física Teórica de la Materia Condensada and Condensed Matter Physics Center (IFIMAC), Universidad Autónoma de Madrid, E28049 Madrid, Spain; Donostia International Physics Center (DIPC), E-20018 Donostia/San Sebastián, Spain; ○ orcid.org/0000-0003-4354-0982; Email: fj.garcia@ uam.es

\section{Authors}

Dongxing Zhao - School of Physical Science and Technology, Southwest University, Chongqing 400715, China; Departamento de Física Teórica de la Materia Condensada and Condensed Matter Physics Center (IFIMAC), Universidad Autónoma de Madrid, E-28049 Madrid, Spain; (1) orcid.org/0000-0002-1110-8805

Rui E. F. Silva - Departamento de Física Teórica de la Materia Condensada and Condensed Matter Physics Center (IFIMAC), Universidad Autónoma de Madrid, E-28049 Madrid, Spain

Clàudia Climent - Departamento de Física Teórica de la Materia Condensada and Condensed Matter Physics Center (IFIMAC), Universidad Autónoma de Madrid, E-28049 Madrid, Spain; O orcid.org/0000-0002-5302-1660

Johannes Feist - Departamento de Física Teórica de la Materia Condensada and Condensed Matter Physics Center (IFIMAC), Universidad Autónoma de Madrid, E-28049 Madrid, Spain; 이이이.org/0000-0002-7972-0646

Antonio I. Fernández-Domínguez - Departamento de Física Teórica de la Materia Condensada and Condensed Matter Physics Center (IFIMAC), Universidad Autónoma de 
Madrid, E-28049 Madrid, Spain; ๑ orcid.org/0000-00028082-395X

Complete contact information is available at:

https://pubs.acs.org/10.1021/acsphotonics.0c01095

\section{Notes}

The authors declare no competing financial interest.

\section{ACKNOWLEDGMENTS}

This work has been funded by the National Natural Science Foundation of China under Grant No. 11804283, by the European Research Council through Grant ERC-2016-STG714870 , and by the Spanish Ministry for Science and Innovation - AEI Grants RTI2018-099737-B-I00, PCI2018093145 (through the QuantERA program of the European Commission), and CEX2018-000805-M (through the "Maria de Maeztu" Programme for Units of Excellence in R\&D). A.I.F.-D. acknowledges support from a 2019 Leonardo Grant for Researchers and Cultural Creators, BBVA Foundation. D.Z. acknowledges financial support from the China Scholarship Council to fund his stay at Universidad Autónoma de Madrid as a postdoctoral fellow.

\section{REFERENCES}

(1) Purcell, E. M. Spontaneous Emission Probabilities at Radio Frequencies. Phys. Rev. 1946, 69, 674.

(2) Yablonovitch, E. Inhibited Spontaneous Emission in Solid-State Physics and Electronics. Phys. Rev. Lett. 1987, 58, 2059.

(3) Pelton, M. Modified spontaneous emission in nanophotonic structures. Nat. Photonics 2015, 9, 427-435.

(4) Akselrod, G. M.; Argyropoulos, C.; Hoang, T. B.; Ciracì, C.; Fang, C.; Huang, J.; Smith, D. R.; Mikkelsen, M. H. Probing the mechanisms of large Purcell enhancement in plasmonic nanoantennas. Nat. Photonics 2014, 8, 835-840.

(5) Novotny, L.; van Hulst, N. Antennas for light. Nat. Photonics 2011, 5, 83-90.

(6) Giannini, V.; Fernández-Domínguez, A. I.; Heck, S. C.; Maier, S. A. Plasmonic Nanoantennas: Fundamentals and Their Use in Controlling the Radiative Properties of Nanoemitters. Chem. Rev. 2011, 111, 3888-3912.

(7) Belacel, C.; Habert, B.; Bigourdan, F.; Marquier, F.; Hugonin, J.P.; Michaelis de Vasconcellos, S.; Lafosse, X.; Coolen, L.; Schwob, C.; Javaux, C.; Dubertret, B.; Greffet, J.-J.; Senellart, P.; Maitre, A. Controlling Spontaneous Emission with Plasmonic Optical Patch Antennas. Nano Lett. 2013, 13, 1516-1521.

(8) Rakovich, A.; Albella, P.; Maier, S. A. Plasmonic control of radiative properties of semiconductor quantum dots coupled to plasmonic ring cavities. ACS Nano 2015, 9, 2648-2658.

(9) Andersen, S. K. H.; Kumar, S.; Bozhevolnyi, S. I. Ultrabright Linearly Polarized Photon Generation from a Nitrogen Vacancy Center in a Nanocube Dimer Antenna. Nano Lett. 2017, 17, 38893895.

(10) Bogdanov, S. I.; Shalaginov, M. Y.; Lagutchev, A. S.; Chiang, C.-C.; Shah, D.; Baburin, A. S.; Ryzhikov, I. A.; Rodionov, I. A.; Kildishev, A. V.; Boltasseva, A.; Shalaev, V. M. Ultrabright RoomTemperature Sub-Nanosecond Emission from Single NitrogenVacancy Centers Coupled to Nanopatch Antennas. Nano Lett. 2018, 18, 4837-4844.

(11) Kaminer, I.; Kooi, S. E.; Shiloh, R.; Zhen, B.; Shen, Y.; López, J. J.; Remez, R.; Skirlo, S. A.; Yang, Y.; Joannopoulos, J. D.; Arie, A.; Soljacic, M. Spectrally and Spatially Resolved Smith-Purcell Radiation in Plasmonic Crystals with Short-Range Disorder. Phys. Rev. X 2017, 7, 011003.

(12) Martín-Jiménez, A.; Fernández-Domínguez, A. I.; Lauwaet, K.; Granados, D.; Miranda, R.; García-Vidal, F. J.; Otero, R. Unveiling the radiative local density of optical states of a plasmonic nanocavity by STM. Nat. Commun. 2020, 11, 1021.

(13) Anger, P.; Bharadwaj, P.; Novotny, L. Enhancement and Quenching of Single-Molecule Fluorescence. Phys. Rev. Lett. 2006, 96, 113002 .

(14) Kühn, S.; Håkanson, U.; Rogobete, L.; Sandoghdar, V. Enhancement of Single-Molecule Fluorescence Using a Gold Nanoparticle as an Optical Nanoantenna. Phys. Rev. Lett. 2006, 97, 017402 .

(15) Muskens, O. L.; Giannini, V.; Sánchez-Gil, J. A.; Gómez Rivas, J. Strong Enhancement of the Radiative Decay Rate of Emitters by Single Plasmonic Nanoantennas. Nano Lett. 2007, 7, 2871-2875.

(16) Kinkhabwala, A.; Yu, Z.; Fan, S.; Avlasevich, Y.; Müllen, K.; Moerner, W. E. Large single-molecule fluorescence enhancements produced by a bowtie nanoantenna. Nat. Photonics 2009, 3, 654-657.

(17) Acuna, G. P.; Möller, F. M.; Holzmeister, P.; Beater, S.; Lalkens, B.; Tinnefeld, P. Fluorescence Enhancement at Docking Sites of DNA-Directed Self-Assembled Nanoantennas. Science 2012, 338, 506-510.

(18) Le Ru, E. C.; Etchegoin, P. G.; Grand, J.; Félidj, N.; Aubard, J.; Lévi, G. Mechanisms of Spectral Profile Modification in SurfaceEnhanced Fluorescence. J. Phys. Chem. C 2007, 111, 16076-16079.

(19) Ringler, M.; Schwemer, A.; Wunderlich, M.; Nichtl, A.; Kürzinger, K.; Klar, T. A.; Feldmann, J. Shaping Emission Spectra of Fluorescent Molecules with Single Plasmonic Nanoresonators. Phys. Rev. Lett. 2008, 100, 203002.

(20) Chizhik, A.; Schleifenbaum, F.; Gutbrod, R.; Chizhik, A.; Khoptyar, D.; Meixner, A. J.; Enderlein, J. Tuning the Fluorescence Emission Spectra of a Single Molecule with a Variable Optical Subwavelength Metal Microcavity. Phys. Rev. Lett. 2009, 102, 073002.

(21) Dong, Z. C.; Zhang, X. L.; Gao, H. Y.; Luo, Y.; Zhang, C.; Chen, L. G.; Zhang, R.; Tao, X.; Zhang, Y.; Yang, J. L.; Hou, J. G. Generation of molecular hot electroluminescence by resonant nanocavity plasmons. Nat. Photonics 2010, 4, 50-54.

(22) Ramezani, M.; Le-Van, Q.; Halpin, A.; Gómez Rivas, J. Nonlinear Emission of Molecular Ensembles Strongly Coupled to Plasmonic Lattices with Structural Imperfections. Phys. Rev. Lett. 2018, 121, 243904.

(23) Wang, D.; Kelkar, H.; Martin-Cano, D.; Rattenbacher, D.; Shkarin, A.; Utikal, T.; Götzinger, S.; Sandoghdar, V. Turning a molecule into a coherent two-level quantum system. Nat. Phys. 2019, $15,483-489$.

(24) Deshmukh, R.; Marques, P.; Panda, A.; Sfeir, M. Y.; Forrest, S. R.; Menon, V. M. Modifying the Spectral Weights of Vibronic Transitions via Strong Coupling to Surface Plasmons. ACS Photonics 2020, 7, 43-48.

(25) Saemisch, L.; Liebel, M.; van Hulst, N. F. Control of Vibronic Transition Rates by Resonant Single-Molecule-Nanoantenna Coupling. Nano Lett. 2020, 20, 4537-4542.

(26) Bellessa, J.; Bonnand, C.; Plenet, J. C.; Mugnier, J. Strong Coupling between Surface Plasmons and Excitons in an Organic Semiconductor. Phys. Rev. Lett. 2004, 93, 036404.

(27) Shi, L.; Hakala, T. K.; Rekola, H. T.; Martikainen, J.-P.; Moerland, R. J.; Törmä, P. Spatial Coherence Properties of Organic Molecules Coupled to Plasmonic Surface Lattice Resonances in the Weak and Strong Coupling Regimes. Phys. Rev. Lett. 2014, 112, 153002 .

(28) Sanvitto, D.; Kéna-Cohen, S. The road towards polaritonic devices. Nat. Mater. 2016, 15, 1061-1073.

(29) Chikkaraddy, R.; De Nijs, B.; Benz, F.; Barrow, S. J.; Scherman, O. A.; Rosta, E.; Demetriadou, A.; Fox, P.; Hess, O.; Baumberg, J. J. Single-molecule strong coupling at room temperature in plasmonic nanocavities. Nature 2016, 535, 127-130.

(30) Liu, R.; Zhou, Z.-K.; Yu, Y.-C.; Zhang, T.; Wang, H.; Liu, G.; Wei, Y.; Chen, H.; Wang, X.-H. Strong Light-Matter Interactions in Single Open Plasmonic Nanocavities at the Quantum Optics Limit. Phys. Rev. Lett. 2017, 118, 237401. 
(31) Neuman, T.; Aizpurua, J. Origin of the asymmetric light emission from molecular exciton-polaritons. Optica 2018, 5, 12471255.

(32) Ojambati, O. S.; Chikkaraddy, R.; Deacon, W. D.; Horton, M.; Kos, D.; Turek, V. A.; Keyser, U. F.; Baumberg, J. J. Quantum electrodynamics at room temperature coupling a single vibrating molecule with a plasmonic nanocavity. Nat. Commun. 2019, 10, 1049.

(33) May, V.; Kühn, O. Charge and Energy Transfer Dynamics in Molecular Systems, 3rd ed.; Wiley-VCH Verlag GmbH \& Co. KGaA: Weinheim, Germany, 2011.

(34) Enderlein, J. Spectral properties of a fluorescing molecule within a spherical metallic nanocavity. Phys. Chem. Chem. Phys. 2002, 4, 2780-2786.

(35) Reitz, M.; Sommer, C.; Genes, C. Langevin Approach to Quantum Optics with Molecules. Phys. Rev. Lett. 2019, 122, 203602.

(36) Maguire, H.; Iles-Smith, J.; Nazir, A. Environmental Nonadditivity and Franck-Condon physics in Nonequilibrium Quantum Systems. Phys. Rev. Lett. 2019, 123, 093601.

(37) Kansanen, K. S. U.; Asikainen, A.; Toppari, J. J.; Groenhof, G.; Heikkilä, T. T. Theory for the stationary polariton response in the presence of vibrations. Phys. Rev. B: Condens. Matter Mater. Phys. 2019, 100, 245426.

(38) Wang, S.; Scholes, G. D.; Hsu, L.-Y. Quantum dynamics of a molecular emitter strongly coupled with surface plasmon polaritons: A macroscopic quantum electrodynamics approach. J. Chem. Phys. 2019, $151,014105$.

(39) Zhang, B.; Liang, W. The vibronic absorption spectra and exciton dynamics of plasmon-exciton hybrid systems in the regimes ranged from Fano antiresonance to Rabi-like splitting. J. Chem. Phys. 2020, 152, 014102.

(40) Schröder, F. A. Y. N.; Chin, A. W. Simulating open quantum dynamics with time-dependent variational matrix product states: Towards microscopic correlation of environment dynamics and reduced system evolution. Phys. Rev. B: Condens. Matter Mater. Phys. 2016, 93, 075105.

(41) del Pino, J.; Schröder, F. A. Y. N.; Chin, A. W.; Feist, J.; GarciaVidal, F. J. Tensor Network Simulation of Non-Markovian Dynamics in Organic Polaritons. Phys. Rev. Lett. 2018, 121, 227401.

(42) Climent, C.; Casanova, D. Electronic structure calculations for the study of D- $\pi$-A organic sensitizers: Exploring polythiophene linkers. Chem. Phys. 2013, 423, 157-166.

(43) Holstein, T. Studies of polaron motion: Part I. The molecularcrystal model. Ann. Phys. 1959, 8, 325-342.

(44) Aspelmeyer, M.; Kippenberg, T. J.; Marquardt, F. Cavity optomechanics. Rev. Mod. Phys. 2014, 86, 1391-1452.

(45) Scheel, S.; Buhmann, S. Y. Macroscopic QED - concepts and applications. Acta Phys. Slovaca 2008, 58, 675-809.

(46) Feist, J.; Fernández-Domínguez, A. I.; García-Vidal, F. J. Macroscopic QED for quantum nanophotonics: Emitter-centered modes as a minimal basis for multi-emitter problems. Nanophotonics 2020.

(47) Buhmann, S. Y.; Welsch, D.-G. Casimir-Polder forces on excited atoms in the strong atom-field coupling regime. Phys. Rev. A: At., Mol., Opt. Phys. 2008, 77, 012110.

(48) Schollwöck, U. The density-matrix renormalization group in the age of matrix product states. Ann. Phys. 2011, 326, 96-192.

(49) Chin, A. W.; Rivas, Á.; Huelga, S. F.; Plenio, M. B. Exact mapping between system-reservoir quantum models and semi-infinite discrete chains using orthogonal polynomials. J. Math. Phys. 2010, 51, 092109.

(50) Chin, A. W.; Huelga, S. F.; Plenio, M. B. Quantum Efficiency in Complex Systems, Part II; Semiconductors and Semimetals; Elsevier, 2011; Vol. 85; pp 115-143.

(51) Orús, R. Tensor networks for complex quantum systems. Nat. Rev. Phys. 2019, 1, 538-550.

(52) Haegeman, J.; Lubich, C.; Oseledets, I.; Vandereycken, B.; Verstraete, F. Unifying time evolution and optimization with matrix product states. Phys. Rev. B: Condens. Matter Mater. Phys. 2016, 94, 165116.
(53) del Pino, J.; Schröder, F. A. Y. N.; Chin, A. W.; Feist, J.; GarciaVidal, F. J. Tensor network simulation of polaron-polaritons in organic microcavities. Phys. Rev. B: Condens. Matter Mater. Phys. 2018, $98,165416$.

(54) Schröder, F. A.; Turban, D. H.; Musser, A. J.; Hine, N. D.; Chin, A. W. Tensor network simulation of multi-environmental open quantum dynamics via machine learning and entanglement renormalisation. Nat. Commun. 2019, 10, 1062.

(55) Yanai, T.; Tew, D. P.; Handy, N. C. A new hybrid exchangecorrelation functional using the Coulomb-attenuating method (CAMB3LYP). Chem. Phys. Lett. 2004, 393, 51-57.

(56) Duschinsky, F. The Importance of the Electron Spectrum inMulti Atomic Molecules. Concerning the Franck-Condon Principle. Acta Physicochim. URSS 1937, 7, 551.

(57) Frisch, M. J.; Trucks, G. W.; Schlegel, H. B.; Scuseria, G. E.; Robb, M. A.; Cheeseman, J. R.; Scalmani, G.; Barone, V.; Petersson, G.; Nakatsuji, H.; et al. Gaussian 16, Revision A.03; Gaussian, 2016.

(58) Palik, E. D. Handbook of Optical Constants of Solids; Academic Press: Burlington, 1998.

(59) Liu, M.; Lee, T.-W.; Gray, S. K.; Guyot-Sionnest, P.; Pelton, M. Excitation of Dark Plasmons in Metal Nanoparticles by a Localized Emitter. Phys. Rev. Lett. 2009, 102, 107401.

(60) Li, R.-Q.; Hernángomez-Pérez, D.; García-Vidal, F. J.; Fernández-Domínguez, A. I. Transformation Optics Approach to Plasmon-Exciton Strong Coupling in Nanocavities. Phys. Rev. Lett. 2016, 117, 107401.

(61) Silva, R. E. F.; del Pino, J.; García-Vidal, F. J.; Feist, J. Polaritonic molecular clock for all-optical ultrafast imaging of wavepacket dynamics without probe pulses. Nat. Commun. 2020, $11,1423$.

(62) Clear, C.; Schofield, R. C.; Major, K. D.; Iles-Smith, J.; Clark, A. S.; McCutcheon, D. P. S. Phonon-Induced Optical Dephasing in Single Organic Molecules. Phys. Rev. Lett. 2020, 124, 153602.

(63) Reitz, M.; Sommer, C.; Gurlek, B.; Sandoghdar, V.; MartinCano, D.; Genes, C. Molecule-photon interactions in phononic environments. Phys. Rev. Research 2020, 2, 033270. 